politische Veränderungen in ihren Heimatstaaten hinarbeiten. Die Unterstützung des bewaffneten Kampfes von Befreiungsbewegungen gegen die Apartheid-Regime hält der schwedische Völkerrechtler und ausgewiesene Kenner des Flüchtlingsrechts für völkerrechtsgemäß, im übrigen leitet er aus dem in afrikanischen Verträgen besonders häufig anzutreffenden Subversionsverbot die gewohnheitsrechtliche Pflicht ab, Flüchtlinge nach Möglichkeit im Zaum zu halten.

Einen weiteren interessanten Beitrag liefert schließlich Peter Nobel, der über die Rechtsstellung von Flüchtlingen in afrikanischen Rechtsordnungen berichtet. Nobel erörtert nicht die komplizierten Probleme der Umsetzung von internationalem in nationales Recht, sondern den Inhalt des letzteren. Nur in sechs afrikanischen Staaten (Senegal, Sudan, Botswana, Tanzania, Zambia, Benin) konnte er rechtliche Regelungen über Flüchtlinge feststellen. „Gewohnheitsrechtlich“ "würden allerdings häufig die Angehörigen verwandter Stämme im Nachbarland freundlich aufgenommen: Hier wäre genauer nach dem Unterschied von Recht und Normen traditioneller Gastfreundschaft zu fragen. Zuzustimmen ist dem Autor, daß eine Verbesserung des Flüchtlingselends, soweit sie mit juristischen Mitteln möglich ist, in der Sphäre des nationalen Rechts ansetzen muß. Zur Erarbeitung von Modellen, die hierfür einen Weg weisen, kann die Rechtswissenschaft noch viel beitragen. Eine Orientierung für diese Aufgabe liefern die engagiert geschriebenen Beiträge dieses sympathischen kleinen $\mathrm{Bu}$ ches.

Philip Kunig

Jorg MANFred Móssner

\title{
Einführung in das Völkerrecht
}

Verlag C. H. Beck, München, 1977, 246 Seiten, 29,80 DM

Das vorliegende Buch ist das dritte einer Reihe von Einführungen in das Völkerrecht, die von Kimminich ${ }^{1}$ und von Weber/v. Wedel ${ }^{\mathbf{2}}$ begonnen wurde. Doch beabsichtigt Mössner keineswegs, sich nur auf ausgetretenen Pfaden zu bewegen. Wollen Weber/v. Wedel dem Leser ,,ein Grundwissen im geltenden Völkerrecht vermitteln, das dazu befähigt, Zusammenhänge der internationalen Politik selbst zu erkennen und sich eine eigene Meinung zu bilden“, und will Kimminich ,,Verständnis wecken, Grundwissen vermitteln, zum Nachdenken und weiteren Studium anregen“, so setzt sich Mössner ,,eine problemorientierte Hinführung zu den tragenden Grundlagen und nicht einen grundrißartigen Ubberblick über das gesamte Rechtsgebiet“" zur Aufgabe. Die Einführung ins Völkerrecht ist in zwei Hauptteile gegliedert. Unter Grundlagen und Strukturen werden Abgrenzungen des Völkerrechts von anderen Rechtsgebieten, rechtstheoretische Grundlagen, Quellen sowie die einzelnen Völkerrechtssubjekte behandelt. Im zweiten Hauptteil befaßt sich Mössner mit den einzelnen völkerrechtlichen Rechtsinstituten, die er unter der Uberschrift ,,Die Aufgaben der Völkerrechtsordnung“ in die drei Gesichtspunkte „Friedensordnung“, ,FFreiheitsordnung“ und „Sozialordnung“ einteilt. Der ,Friedensordnung“ werden das Vertragsrecht, das Diplomatenrecht, das Deliktsrecht, die friedliche Streiterledigung, das Gewaltverbot, das Recht der kollektiven Friedenssicherung und das Kriegsrecht zugeordnet. Die „,Freiheitsordnung“ wird durch die Kapitel über die Grundrechte der Staaten und die individuellen Rechtspositionen beschrieben. Schließlich fällt unter die Aufgabe der ,Sozialordnung“ das Seerecht, das Luft- und Weltraumrecht sowie das internationale Umweltschutzrecht.

1 Kimminich, Einführung in das Völkerrecht, Pullach bei München, 1975.

2 Weber/v. Wedel, Grundkurs Völkerrecht, Frankfurt/Main, 1977 - vgl. dazu die Besprechung von Kunig in VRU, 1978, S. 130 ff. 
Mit dieser Zusammenfassung der völkerrechtlichen Rechtsinstitute will Mössner gegenüber anderen Völkerrechtsdarstellungen in besonderer Weise deren Grundgedanken deutlich herausheben, die ,,vielmehr verschiedene Aspekte des großen Generalthemas des Rechts: der Gerechtigkeit" sind. Zwar lassen sich völkerrechtliche Darstellungen nach den verschiedensten Gesichtspunkten einteilen, doch leistet Mössner dem Ansehen und damit letztlich auch der Durchsetzbarkeit des Völkerrechts keinen guten Dienst, wenn er als Aufgabe des Völkerrechts die Erreichung eines gerechten Zustandes erwarten läßt. Solche Erwartungen können nur enttäuscht werden, denn dem Völkerrecht kann als Koordinationsrecht keine eigenständige Funktion gegenüber der Politik zukommen. Wie sehr hier eine Auseinandersetzung mit dem Verhältnis Völkerrecht - Politik zu vermissen ist, wird auch hinsichtlich eines anderen Aspekts der „Friedensordnung“ deutlich: Was für ein Frieden oder wessen Frieden soll erhalten werden? Im Zeitalter der Supermächte mit ihren Hegemonialsphären kann Frieden allenfalls als Nicht-Krieg bezeichnet werden, und das Problem der Gerechtigkeit bleibt einer Lösung unzugänglich. Der zweifelhafte Nutzen der angeführten Einteilung läßt es weiterhin unverständlich erscheinen, warum einerseits Entstehung und rechtliche Wirkung von Völkergewohnheitsrecht unter den Hauptteil ,,Grundlagen und Struktur“ “fallen, andererseits aber nur die rechtliche Wirkung von Verträgen diesem Punkt zugeordnet wird, während die Entstehung und Auslegung von völkerrechtlichen Verträgen unter den Hauptteil „Aufgaben“ und das Kapitel ,,Herstellung einer internationalen Kommunikationsordnung" fallen. Bedarf nicht die Entstehung von Völkergewohnheitsrecht ebenso der internationalen Kommunikation - wenn auch nicht in derart förmlicher Weise wie vom Vertragsrecht -, besonders wenn man die Bedeutung des Vertrauensschutzes gebührend berücksichtigt?

Der Inhalt der Darstellung gibt nur wenig Anlaß zur Kritik. Die Meinung, daß einer internationalen Konvention über die Einführung einer 200 Seemeilen breiten Wirtschaftszone auf der Hohen See ,,ein besonderes Element der Rechtsschöpfung von objektiver Natur innewohnt" (S. 29), verwischt die Unterschiede zwischen normativer Bindung und unvermeidlicher faktischer Gebundenheit. Nichtteilnehmer der Konvention können nicht gegen ihren Willen an die Konvention gebunden werden, es sei denn eine Bindung träte nach den Grundsätzen von aquiescence und estoppel ein. Auffällig ist die spärliche Behandlung der Möglichkeiten zur Lösung der Konflikte zwischen Völkerrechteinerseits und Landesrecht (S. 55-58) wie europäischem Gemeinschaftsrecht (S. 51/52) andererseits. Gerade hierauf hätte auch im Rahmen einer Einführung größeres Augenmerk gelegt werden sollen, weil ohne die Kenntnis dieser Zusammenhänge nicht über wesentliche Bereiche der Durchsetzung und Anwendung von Völkerrecht geurteilt werden kann. Bis auf zwei unbedeutende Erwähnungen (S. 84 und S. 146) fehlt eine Befassung mit der sozialistischen Völkerrechtstheorie. Dies wäre zumindest in dem Maße erforderlich, in dem auf die voluntaristischen, normativistischen, naturrechtlichen, anthropologischen und soziologischen Theorien eingegangen wird.

Auf die Einarbeitung von Fußnoten wurde verzichtet, sondern den einzelnen Kapiteln eine begrenzte Auswahl weiterführender Literatur vorangestellt. Zur Unterstreichung seiner Ausführungen bedient sich Mössner einer Vielzahl von Beispielen und Fällen internationaler Gerichte. Er geht dabei auf Ablauf und Entscheidung ein, ohne daß es zu einer Ubergewichtung kommt. Die Darlegungen sind daher leicht lesbar und werden dem begrenzten Zweck einer Einführung vollauf gerecht. 